\title{
BMJ Open Prospective observational cohort study of factors influencing trial participation in people with motor neuron disease (FIT-participation-MND): a protocol
}

To cite: Beswick E, Glasmacher SA, Dakin R, et al. Prospective observational cohort study of factors influencing trial participation in people with motor neuron disease (FIT-participationMND): a protocol. BMJ Open 2021;11:e044996. doi:10.1136/ bmjopen-2020-044996

- Prepublication history for this paper is available online To view these files, please visit the journal online (http://dx.doi org/10.1136/bmjopen-2020044996).

Received 18 September 2020 Revised 31 December 2020 Accepted 14 January 2021

Check for updates

(C) Author(s) (or their employer(s)) 2021. Re-use permitted under CC BY-NC. No commercial re-use. See rights and permissions. Published by BMJ.

For numbered affiliations see end of article.

Correspondence to

Dr Suvankar Pal;

suvankar.pal@ed.ac.uk

\section{ABSTRACT}

Introduction Motor neuron disease (MND) is a rapidly progressive and fatal neurodegenerative disorder with limited treatment options. The Motor Neuron Disease Systematic Multi-Arm Randomised Adaptive Trial (MNDSMART) is a multisite UK trial seeking to address the paucity in effective disease-modifying drugs for people with MND (pwMND). Historically, neurological trials have been plagued by suboptimal recruitment and high rates of attrition. Failure to recruit and/or retain participants can cause insufficiently representative samples, terminated trials or invalid conclusions. This study investigates patient-specific factors affecting recruitment and retention of pwMND to MND-SMART. Improved understanding of these factors may improve trial protocol design, optimise recruitment and retention.

Methods and analysis PwMND on the Scottish MND Register, Clinical Audit Research and Evaluation of MND (CARE-MND), will be invited to participate in a prospective observational cohort study that investigates factors affecting trial participation and attrition. We hypothesise that patient-specific factors will significantly affect trial recruitment and retention. Participants will complete the Hospital Anxiety and Depression Scale, 9-Item Patient Health Questionnaire and State-Trait Anxiety InventoryForm $Y$ to evaluate neuropsychiatric symptoms, the ALS-Specific Quality of Life Questionnaire-Brief Form and Centre for Disease Control and Prevention-HealthRelated Quality of Life for quality of life and a novel study-specific questionnaire on Attitudes towards Clinical Trial Participation (ACT-Q). Clinical data on phenotype, cognition (Edinburgh Cognitive and Behavioural ALS Screen) and physical functioning (Amyotrophic Lateral Sclerosis Functional Rating Scale-Revised) will also be collated. Caregivers will complete the Brief Dimensional Apathy Scale. After 12 months, a data request to MNDSMART will evaluate recruitment and retention. Descriptive statistics will summarise and compare assessments and participants reaching impairment thresholds. Variable groupings: attitudes, quality of life, cognition, behaviour, physical functioning, neuropsychiatric and phenotype. Univariate and multivariable logistic regression will explore association with participation/withdrawal in MND-SMART; presented as 0 Rs and $95 \%$ Cls.
Strengths and limitations of this study

- The first observational cohort study prospectively assessing factors influencing people with motor neuron disease to participate, and remain in, a clinical trial.

- Better understanding of factors that affect recruitment and retention can inform future trial design.

- Impossible to account for all potential influences on the variability in human behaviour.

- As the population is Scotland-based, there may be additional barriers to participation or factors affecting attrition in trial sites across the UK.

Ethics and dissemination Ethical approval was provided by the West of Scotland Research Ethics Committee 3 (20/WS/0067) on 12 May 2020. The results of this study will be published in a peer-reviewed journal, presented at academic conferences and disseminated to participants and the public.

\section{INTRODUCTION}

Clinical trials in motor neuron disease

Motor neuron disease (MND) is a rapidly progressive, incurable and uniformly fatal neurodegenerative disorder. Mean age of onset is 65.3 years and only $51.3 \%$ of people with MND (pwMND) survive more than 12 months from diagnosis. ${ }^{1}$ The disorder has a significant impact on multiple aspects of an individual's life necessitating a holistic approach to clinical care and trial design.

There is an urgent need for new therapies in MND. Only one disease-modifying therapy, riluzole, has been approved for treatment in the UK, with limited impact on median survival. ${ }^{2}$ The recently published Airlie House guidelines encapsulate the new direction of trials in this area; with clear recommendations to rethink outcome measures, stratify participant characteristics and use academic 
consensus and historical trial findings to inform future design. ${ }^{3}$

\section{Recruitment and retention in MND trials}

The accurate identification of factors that impact on recruitment and retention of participants in research studies is important when considering trial design. ${ }^{4}$ Recruitment should involve selection of participants representative of the target population, in numbers sufficient to fulfil trial-specific power calculations. Previously, while restrictive inclusion criteria have been advantageous to stratify a heterogeneous population to detect an effect, results from these studies may not be readily generalisable, and restrict opportunities for research participation. While $83 \%$ of pwMND indicated that they would be open to participating in research trials, ${ }^{5}$ surveyed clinicians estimate enrolment figures of $25 \%$ in trials, primarily due to unsuitability of pwMND within stated inclusion criteria. ${ }^{6}$

Trials have utilised narrow inclusion criteria in an attempt to stratify subgroups, however, this may impact on homogenisation of trial outcomes at the cost of inclusivity. ${ }^{7}$ As MND-SMART (Motor Neuron Disease Systematic Multi-Arm Adaptive Trial) involves broader inclusion criteria than many previous trials, we expect higher rates of enrolment.

Attrition is defined as the loss of participating individuals to follow-up or as a result of missing data at one of more time-points. ${ }^{8}$ While some attrition is inevitable, ensuring optimal retention is an important consideration in trial design. Clinical trials in pwMND frequently report attrition rates over $20 \% ;^{910}$ risk of bias is high at attrition rates in this threshold. ${ }^{11}$ Any level of attrition may result in bias in results reported as the characteristics of those individuals remaining, versus those lost to follow-up or with significant levels of missing data, may differ.

Suboptimal recruitment and retention can affect a study's power, in turn significantly impacting conclusions. ${ }^{12}$ These methodological issues can lead to trials reporting invalid or inconclusive results, prolonged trial times and potential premature termination. ${ }^{4} 13$ Further investigation of factors that may account for variability in recruitment and retention, particularly within $\mathrm{MND}$, is essential to devise strategies to address issues in enrolment and attrition and improve trial delivery.

\section{New directions in MND trials}

MND-SMART is a multisite UK clinical trial, which seeks to evaluate the effects of repurposed medicines with potential neuroprotective properties. Full details of the trial design and selection criteria are available at clinicaltrials. gov (NCT04302870) and EudraCT (Trial record number: 2019-000099-41). MND-SMART is a long-running trial, expected to evaluate several candidate repurposed drugs over the next two decades under a single umbrella protocol. Currently, memantine and trazodone are being evaluated against placebo.

Primary objectives are to assess the impact of these candidate drugs on functional ability, as measured using the Amyotrophic Lateral Sclerosis Functional Rating Scale-Revised (ALS-FRS(R)), ${ }^{14}$ and also on survival. Secondary outcomes include the impact of these medicines on cognition, respiratory functioning, affective symptoms and quality of life in pwMND.

The multiarm, multistage, adaptive design has been shown to be particularly beneficial in enabling a reduction of patient numbers and time required to test more than one candidate drug in later stage trials of stroke ${ }^{15}$ and cancer ${ }^{16}$. These may be particularly crucial changes in trial delivery for rarer and higher-burden diseases such as MND. Broad inclusion criteria intend to promote participation and ensure that the trial is available to a large number of pwMND, ultimately intending to capture the heterogeneity of this condition and improve the generalisability of findings. Establishing multiple stages with predefined interim analysis also reduces the chance of a patient taking an ineffective drug for longer than necessary, crucial in a condition with such a short life expectancy.

\section{Rationale}

A review of clinical trials in oncology ${ }^{17}$ identified three areas that impact on recruitment: patient factors, trial factors and doctor factors. This concept was also reflected in Atassi' $\mathrm{s}^{5}$ review of factors affecting adherence in MND trials; study population characteristics, trial design and site/staff facilities. MND-SMART seeks to address many trial factors such as inclusive trial participant criteria, remote follow-up appointments to address progressive disability and liquid medication to minimise potential swallowing difficulties.

In MND-SMART, many of the trial and doctor-specific factors that have previously affected engagement with MND trials have been addressed. This provides us with a unique opportunity to explore how patient-specific factors can also influence trial participation decisions. Although focused on a single trial, we believe these findings will inform future trial design, promote inclusivity and support trial teams in retaining participants.

The presence of neuropsychiatric conditions, behaviour change and cognitive impairment pose significant challenges for recruitment and can impact on a person's ability to give informed consent and adhere to study protocol. ${ }^{518}$ Participants' demographic characteristics ${ }^{19} 20$ and attitudes towards research and health behaviours ${ }^{21}$ may also be predictive of trial enrolment and attrition.

However, we currently have no knowledge of how prevalent and impactful these patient-specific factors are on pwMND and their decision to participate and remain in a clinical trial. This study will seek to address this knowledge gap, exploring what factors define the trial 'participant' and are associated with retention at follow-up.

\section{Aims and hypothesis}

This prospective observational cohort study will investigate how patient-specific factors impact on recruitment 
and retention within the context of the first multi-arm multicentre UK clinical trial in MND.

\section{Aims}

1. Evaluate the characteristics of individuals who do, or do not, participate in MND-SMART

2. Compare the characteristics of FIT-P-MND participants who remain enrolled on MND-SMART after 12 months and those who are lost to follow-up

\section{Hypothesis}

We hypothesise that patient-specific factors, such as neuropsychiatric symptoms, cognitive impairment, behavioural change, phenotype, quality of life and physical functioning will significantly impact on pwMND's decision to participate, and remain in, MND-SMART.

\section{METHODS}

\section{Study design}

This prospective observational cohort study will evaluate how patient-specific factors in pwMND affect their participation in MND-SMART, a multi-arm UK-wide clinical trial.

Using a range of assessments this study will evaluate patient-specific factors in pwMND at a specific point in time: neuropsychiatric symptoms (specifically depression, anxiety and suicidality), apathy, attitudes to clinical trials and quality of life. This will be supplemented with clinical care data and trial participation data derived from the individual's Clinical Audit Research and Evaluation of MND (CARE-MND) register record, and MND-SMART data if they choose to also participate in the trial.

After 12 months, we will use an additional data request to MND-SMART to explore which of the FITP-MND participants also joined the trial, and of these, who remains enrolled in the trial after 12 months. This study will explore how the patient-specific factors, evaluated through questionnaires and clinical data, impacted on the recruitment to, and retention of, this cohort of pwMND to MND-SMART.

\section{CARE-MND register}

The FIT-P-MND project will use CARE-MND to facilitate recruitment, access clinicaldata for pwMND living in Scotland.

Established in 1989, the Scottish Motor Neuron Disease Register was the first population-based register for MND in the world.$^{22}$ In 2015, the register was relaunched as the electronic platform CARE-MND. This has facilitated detailed longitudinal phenotyping of pwMND in Scotland from diagnosis to death, with allied tissue and brain banks and a research interest register allowing pwMND the ability to register interest in future observational and interventional studies. ${ }^{23}$ The CARE-MND register has 99\% case ascertainment for pwMND in Scotland where there is an incidence of 180-220 new cases a year, and a prevalence of approximately 450 pwMND. ${ }^{23}$ FIT-P-MND will focus on Scottish participants.
Table 1 Overview of FIT-participation-MND study timeline

\begin{tabular}{ll}
\hline Key study aspect & Actual or projected date \\
\hline $\begin{array}{l}\text { Favourable ethical opinion } \\
\text { obtained }\end{array}$ & 12 May 2020 \\
$\begin{array}{l}\text { Site approval to commence } \\
\text { recruitment }\end{array}$ & 8 July 2020 \\
Recruitment commences & 10 August 2020 \\
$\begin{array}{l}\text { Recruitment planned to close } \\
\text { CARE-MND data request }\end{array}$ & March 2021 \\
MND-SMART data request & March 2021 \\
\hline
\end{tabular}

CARE, Clinical Audit Research and Evaluation ; FIT, factors influencing trial; MND, motor neuron disease ; SMART, Systematic Multi-Arm Randomised Adaptive Trial.

\section{Stages of study}

This study will involve three time-points of data collection, with participants directly involved in the first stage only:

1. Questionnaire completion: participant and caregiver questionnaire packs.

2. CARE-MND data request: additional covariates collected in routine clinical care.

3. MND-SMART data request: trial involvement and participation.

\section{Study Timeline}

Timeline for the current study was impacted by the COVID-19 pandemic, as non-COVID research and recruitment was temporarily halted. Table 1 provides an overview for key time points in the study, and projected dates for recruitment and data collection.

\section{Recruitment}

All individuals on the CARE-MND register who have consented to receive information on studies which they may be eligible for will be invited to participate in FITP-MND via a postal invitation pack.

Potential FIT-P-MND participants will receive this postal pack with information on the study and a Consent Form. They will also be asked to identify a caregiver who would be willing to complete a behaviour questionnaire and they will receive a separate Consent Form and Information Sheet.

Participants can indicate on their Consent Form their preferred completion method; online survey, in-person appointment in Edinburgh, postal or telephone. These options have been selected to maximise accessibility and inclusivity.

\section{Sample size considerations}

This study will aim to recruit a sample of 120 individuals with a diagnosis of MND.

The required sample size was determined using the primary research objectives, which will be answered using regression analysis. The calculation is based on the use of a logistical regression model, as recruitment of pwMND 
to the MND-SMART clinical trial is a binary outcome variable (yes/no to participation).

An OR (measure of association between an exposure and an outcome) of 1.70 with power at 0.70 provides a sample size estimate of 111 .

There are around 420 pwMND in Scotland at any point in time, circa 220 of whom have provided consent on CARE-MND to be contacted about research. All participants on the CARE-MND register who are eligible will be invited to participate.

Previous research using postal questionnaires in pwMND reports a responserate of $63 \% .{ }^{24}$ However, we anticipate that adding the options of completion via online survey or telephone will improve response rates. As a result, we expect 120 individuals to be an obtainable sample size, based on a $60 \%$ response rate estimate.

Across all Scottish sites, the MND-SMART projected recruitment is 100 participants per year, with attrition due to death or withdrawal predicted at $20 \%$ annually. We estimate that around $80 \%$ of the 120 participants in FIT-P-MND will also enrol in MND-SMART, 100 people. Based on projected annual attrition rates of $20 \%$ for MND-SMART, we expect 80 of these individuals to remain in MND-SMART 12 months later.

As this is an exploratory study, looking at nine variables (using grouping to simplify analysis and presentation), and a relatively rare condition, sample size is based on the number of potential participants available and descriptive analysis methods will be utilised to explore the data.

\section{Study assessments}

Table 2 includes a summary of all study assessments included in FIT-P-MND participant and caregiver engagement and data requests. The questionnaire set for pwMND will take around 45 minutes, depending on physical decline and speech impairment, and includes ; Hospital Anxiety and Depression Scale, 9-Item Patient Health Questionnaire and State-Trait Anxiety InventoryForm Y. FIT-P-MND participants will also be invited to questionnaires on quality of life: ALS-Specific Quality of Life Questionnaire-Brief Form and Centre for Disease Control and Prevention-Health-Related Quality of Life. Finally, participants will be asked to complete the Attitudes towards Clinical Trial Participation Questionnaire to evaluate attitudes towards trial participation. Caregivers will be invited to complete the Brief Dimensional Apathy Scale to consider behavioural changes of the participant (circa 5 minutes). Questionnaires will take around 50 minutes in total.

Data requests to CARE-MND and MND-SMART for additional data on physical functioning, cognition and clinical phenotype enable us to reduce burden on participants by ensuring brevity in study visits. We will use ALSFRS(R) and Edinburgh Cognitive and Behavioural ALS Screen scores from CARE-MND, or MND-SMART if the individual is also a trial participant, to ensure we collect scores closest to the time-point that the FIT-P-MND study assessments are undertaken.
Table 2 Study assessments, CARE-MND and MNDSMART data requests

\begin{tabular}{ll}
\hline Data source & Name of assessment \\
\hline $\begin{array}{l}\text { Study assessment } \\
\text { questionnaires: FIT-P- }\end{array}$ & ACT-Q (Attitudes towards Clinical \\
MND participant with & Trial Participation Questionnaire) \\
MND & HADS (Hospital Anxiety and \\
& Depression Scale) $)^{27} 28$ \\
& STAI-Y (State-Trait Anxiety \\
& Inventory-Form Y) \\
& PHQ-9 (9-Item Patient Health \\
& Questionnaire) \\
& ALSSQOL-20 (ALS-Specific \\
& Quality of Life Questionnaire-Brief \\
& Form) ${ }^{32}$ \\
& CDC HQOL-4 (Centre for Disease \\
& Control and Prevention-Health- \\
& Related Quality of Life) \\
& b-DAS (Brief Dimensional Apathy \\
& Scale) ${ }^{34} 35$
\end{tabular}

\begin{tabular}{|c|c|}
\hline \multirow{6}{*}{$\begin{array}{l}\text { CARE-MND data } \\
\text { request }\end{array}$} & Clinical phenotype data \\
\hline & Date of diagnosis \\
\hline & - Age at diagnosis \\
\hline & - Classification of MND \\
\hline & S Site of onset \\
\hline & - Family history \\
\hline \multirow[t]{2}{*}{$\begin{array}{l}\text { CARE-MND or MND- } \\
\text { SMART data request }\end{array}$} & $\begin{array}{l}\text { ALS-FRS (R) (Amyotrophic Lateral } \\
\text { Sclerosis Functional Rating Scale- } \\
\text { Revised) }\end{array}$ \\
\hline & $\begin{array}{l}\text { ECAS (Edinburgh Cognitive and } \\
\text { Behavioural ALS Screen) }\end{array}$ \\
\hline \multirow[t]{9}{*}{$\begin{array}{l}\text { MND-SMART data } \\
\text { request }\end{array}$} & $\begin{array}{l}\text { MND-SMART recruitment and } \\
\text { retention data }\end{array}$ \\
\hline & $\begin{array}{l}\text { Date MND-SMART Participant } \\
\text { Information Sheet given }\end{array}$ \\
\hline & Date of screening \\
\hline & Date of randomisation \\
\hline & D Date of withdrawal \\
\hline & $\begin{array}{l}\text { Date of last appointment if } \\
\text { withdrawn }\end{array}$ \\
\hline & $\begin{array}{l}\text { Reason for withdrawal if } \\
\text { provided }\end{array}$ \\
\hline & - Status (alive/deceased) \\
\hline & - Date of death (if applicable) \\
\hline
\end{tabular}

CARE, Clinical Audit Research and Evaluation; MND, motor neuron disease; SMART, Systematic Multi-Arm Randomised Adaptive Trial.

\section{Inclusion/exclusion criteria}

Table 3 summarises the inclusion and exclusion criteria for pwMND and their caregivers who wish to participate in FIT-P-MND. These criteria have been primarily 
Table 3 Criteria for FIT-participation-MND participants and caregivers

\section{For FIT-participation-MND participant with MND}

\begin{tabular}{|c|c|}
\hline $\begin{array}{l}\text { Inclusion } \\
\text { criteria }\end{array}$ & $\begin{array}{l}\text { Over } 18 \\
\text { Confirmed diagnosis of MND (including } \\
\text { the following subtypes: amyotrophic lateral } \\
\text { sclerosis by El Escorial Criteria (possible, } \\
\text { probable and definite), primary lateral } \\
\text { sclerosis and progressive muscular atrophy) } \\
\text { Able to provide informed consent (proxy } \\
\text { signature accepted if limb dysfunction) } \\
\text { Fluent in English }\end{array}$ \\
\hline $\begin{array}{l}\text { Exclusion } \\
\text { criteria }\end{array}$ & $\begin{aligned} & \text { Diagnosis of Frontotemporal Dementia } \\
& \text { (FTD-MND) } \\
& \text { - Unable to provide informed consent } \\
& \text { - Resident outside Scotland }\end{aligned}$ \\
\hline For careg & iver \\
\hline $\begin{array}{l}\text { Inclusion } \\
\text { criteria }\end{array}$ & $\begin{array}{l}\text { Able and willing to complete a brief } \\
\text { questionnaire regarding the participant's } \\
\text { behaviour } \\
\text { - Family member, spouse, relative, friend or } \\
\text { partner for pwMND } \\
\text { Primary caring responsibilities for pwMND } \\
\text { - Fluent in English }\end{array}$ \\
\hline $\begin{array}{l}\text { Exclusion } \\
\text { criteria }\end{array}$ & $\begin{array}{l}\text { Paid carers-excluded to ensure they know the } \\
\text { person pre-MND diagnosis } \\
\text { Not fluent in English } \\
\text { Unable to provide informed consent } \\
\text { Diagnosis of MND }\end{array}$ \\
\hline
\end{tabular}

FIT, factors influencing trial-participation; MND, motor neuron disease.

selected to align with the criteria for MND-SMART to ensure maximum overlap in the two participant groups.

\section{Public and patient involvement statement}

PwMND were first involved in the research when they were emailed the ACT-Q, Participant Information Sheet and Consent Form and asked to provide feedback. PwMND were asked to consider the questionnaire structure of the ACT-Q, provide an estimate of the time taken to complete and asked to suggest any additional factors which may influence their attitudes towards trial participation.

PwMND were invited to provide feedback on Participant Information Sheets and Consent Forms, particularly clarity of study aims, and additional aspects of MND which may potentially affect trial participation. Feedback from these individuals was used to refine the list of exploratory covariates in the study, and particularly the items included on the ACT-Q. Time required to participate in the research was based on feedback from patients, existing time-to-complete data for each of the established questionnaires, with additional time for potential communication or physical difficulties.
All participants in the study, who chose to, will be sent a copy of the study findings. PwMND and their caregivers will be consulted regarding the best way to disseminate findings and provide feedback on initial versions of the study summary documents to ensure clarity of presentation and suitability for this audience.

\section{ANALYSIS PLAN}

The study questionnaires will be grouped into domains to reduce the number of candidate variables in the analysis as shown in table 4 . The total scores of individual questionnaires will be summed into an overall summary score for each domain, which will be included in the subsequent analysis. As all scores represent the same directionality (higher score indicates greater impairment) a summed score will provide an overall indication of the level of impairment for each individual per grouped domain.

We will present the mean scores for each assessment, displayed in the factor groupings discussed above, with ranges and $\mathrm{SD}$.

\section{Scoring the ACT-Q}

The Attitudes towards Clinical Trial Participation Questionnaire (ACT-Q) is a brief trial-specific questionnaire to quantify FIT-P-MND's attitudes towards involvement in research, and multi-arm clinical trials in particular (table 5). Developed by the investigators, based on Kessel's survey $^{25}$ and Ellis' tripartite model ${ }^{17}$ on factors impacting trial engagement.

Each potential response is scored on the participant's rating of its importance to their decision-making process and an overall score for each factor will be produced per individual. Items 6,7 and 13 will be reverse scored, indicating less agreement with the statement.

The final aspect of this questionnaire is evaluating FITP-MND participants' understanding of five key features of design. Respondents will indicate on a 5-point scale to represent level of understanding.

\section{Statistical analysis}

We will use logistic regression to model the impact of the aforementioned independent variables on the FITP-MND participant's decision to enrol or not enrol in MND-SMART. All variables will be considered in univariate and subsequently multivariable analysis. Results will be presented as OR and 95\% CIs.

12 months after the obtaining the questionnaire responses, we will extract participation data from MND-SMART.

As this is an exploratory study with nine categories of covariates, we will not be using an adjusted alpha-level to correct for multitesting. The Bonferroni method of correcting $\mathrm{p}$ values may not be suitable for this analysis as hypotheses are predefined, not all variables must be significant to reject the null hypothesis and descriptive statistics will also be of relevant when interpreting the findings. ${ }^{26}$ 
Table 4 Grouping of exploratory covariates

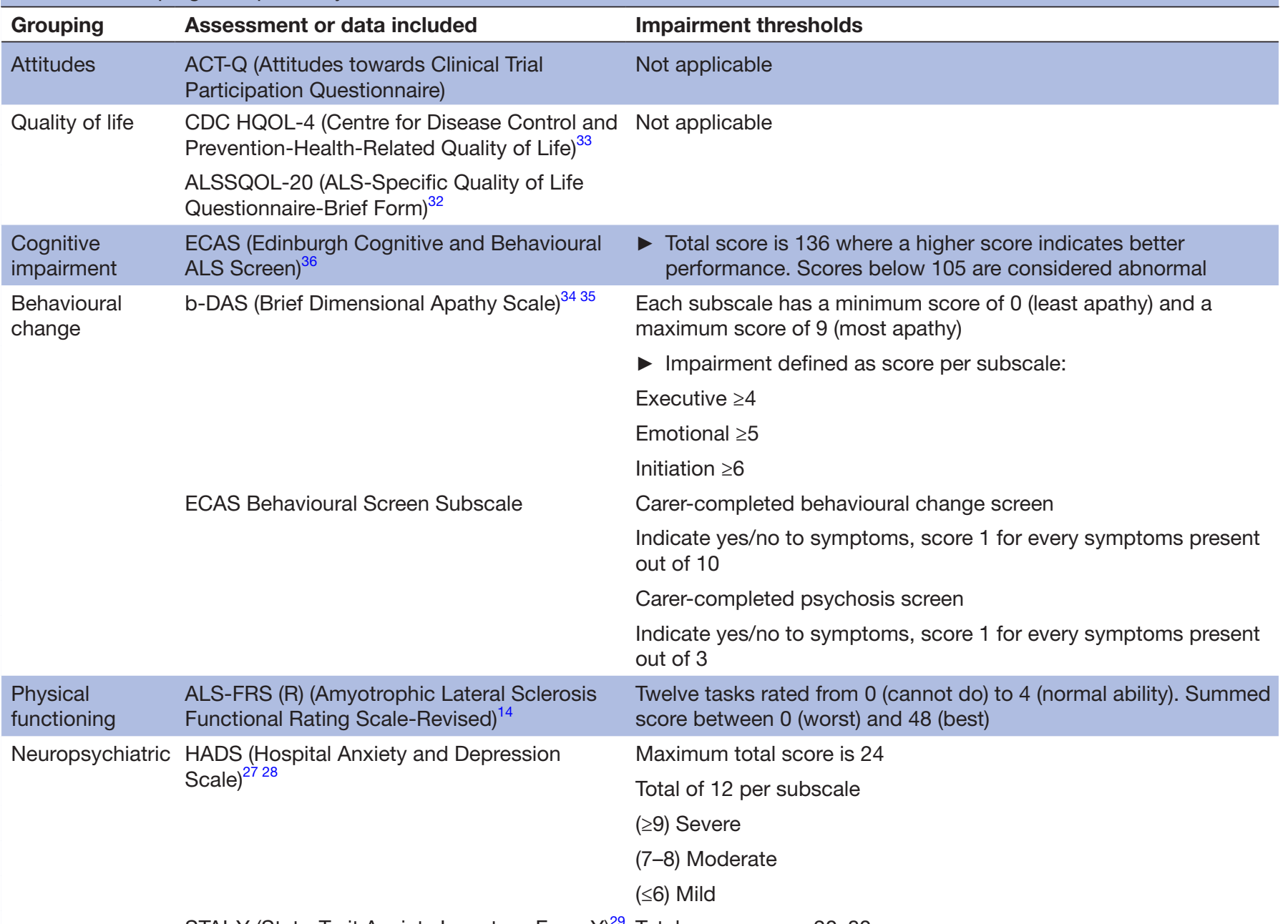

STAI-Y (State-Trait Anxiety Inventory-Form Y) ${ }^{29}$ Total score ranges 20-80

$$
\begin{aligned}
& (\geq 60) \text { High } \\
& (40-59) \text { Moderate } \\
& (20-39) \text { Low }
\end{aligned}
$$

PHQ-9 (9-Item Patient Health Questionnaire) ${ }^{30}$ Depression:

Total score ranges from 0 to 27

(20-27) Severe

(15-19) Moderately severe

(10-14) Moderate

(5-9) Mild

(1-4) Minimal

Suicidality:

Item 9 scores from (0) not at all

1. Several days

2. More than half the days

3. Nearly every day

Scores of $\geq 1$ indicates presence of suicidal ideation

$\begin{array}{lll}\text { Clinical } & \text { MND classification } & \text { Not applicable } \\ \text { phenotype } & \text { Site of onset (spinal, bulbar, pure } & \\ & \text { respiratory) } & \\ & \text { Age at diagnosis }\end{array}$

MND, motor neuron disease. 
Table 5 Items of the Attitudes to Clinical Trials Questionnaire (ACT-Q) and grouping

\begin{tabular}{|c|c|}
\hline Category & Item \\
\hline \multirow[t]{3}{*}{$\begin{array}{l}\text { Practical } \\
\text { burden }\end{array}$} & $\begin{array}{l}\text { 1. The time commitment required to } \\
\text { participate }\end{array}$ \\
\hline & 2. The distance to the clinic is too far \\
\hline & 3. I already feel I have a lot of appointments \\
\hline \multirow[t]{2}{*}{$\begin{array}{l}\text { Disease } \\
\text { burden }\end{array}$} & $\begin{array}{l}4 \text { I would not feel well enough to participate } \\
\text { because of how my condition affects me }\end{array}$ \\
\hline & $\begin{array}{l}\text { 5. I am concerned about the potential } \\
\text { dangers and side effects of trial medications }\end{array}$ \\
\hline \multirow[t]{4}{*}{$\begin{array}{l}\text { Altruistic } \\
\text { motivations }\end{array}$} & $\begin{array}{l}\text { 6. I may not benefit personally from the } \\
\text { development of new drugs }\end{array}$ \\
\hline & $\begin{array}{l}\text { 7. I am worried about the possibility of being } \\
\text { assigned to the placebo group }\end{array}$ \\
\hline & $\begin{array}{l}\text { 8. I want to help other people with the same } \\
\text { condition as me }\end{array}$ \\
\hline & $\begin{array}{l}\text { 9. I want the opportunity to contribute to } \\
\text { research }\end{array}$ \\
\hline \multirow[t]{3}{*}{$\begin{array}{l}\text { Practical } \\
\text { benefits }\end{array}$} & $\begin{array}{l}\text { 10. I will get additional monitoring of how my } \\
\text { condition is changing }\end{array}$ \\
\hline & $\begin{array}{l}\text { 11. I will receive more regular contact with } \\
\text { medical staff }\end{array}$ \\
\hline & $\begin{array}{l}\text { 12. I may get to try new medicines which are } \\
\text { not available to everyone with my condition }\end{array}$ \\
\hline \multirow[t]{2}{*}{$\begin{array}{l}\text { Research } \\
\text { engagement }\end{array}$} & $\begin{array}{l}\text { 13. I am already participating in other } \\
\text { research projects }\end{array}$ \\
\hline & $\begin{array}{l}\text { 14. I have participated in research before and } \\
\text { had a positive experience }\end{array}$ \\
\hline
\end{tabular}

\section{Missing data}

If particular covariates, certain assessments or questionnaires, are not completed fully by the majority of participants, we will consider removing this variable from analysis. Missing data within individual questionnaires will be handled using multiple imputation. Incomplete questionnaires will not be returned to participants. As participants do not require a caregiver to participate, a missing behavioural questionnaire will not be included in thresholds for missing data. The covariate of behavioural change will be included where possible.

\section{MANAGEMENT OF POTENTIAL RISK}

All FIT-P-MND participants, and caregivers, will provide informed consent and acknowledge they have read the study information. Participant-facing documentation highlights the voluntary nature of the study and requirements of participation. There are no direct risks involved in participating, however, as some of the questionnaires focus on mental health we acknowledge this may be distressing, clinical teams will be informed of any significant scores. The individual's GP will also be contacted to inform them of study involvement.
A potential issue is fatigue, which may be induced by the length of assessment administration. Participants will be encouraged to inform the researcher if they are experiencing any and the option to complete at home enables participants who have significant issues with physical and mental fatigue to take breaks and complete the questionnaires at their own pace.

Data management and confidentiality of FIT-P-MND participants will be managed by assigning participant ID codes to anonymise responses. The use of identifiable information will be minimised.

\section{ETHICS AND DISSEMINATION}

This research is co-sponsored by the University of Edinburgh and NHS Lothian. Representatives from the Academic and Clinical Central Office for Research and Development have reviewed and approved this project. Ethical approval was provided by the West of Scotland Research Ethics Committee 3 on 12th May 2020 (REC Reference: 20/WS/0067).

Only pwMND who have provided prior consent to be contacted about ongoing research on their CARE-MND record will be invited to participate. The Anne Rowling Regenerative Neurology Clinic hosts the register, the data processor at the clinic will contact the potential participants' MND nurse prior to posting recruitment packs.

At the end of the study a lay summary will be sent to the participants, or their nominated representative, for individuals who have indicated they would like to receive one on their Consent Form. The results will be disseminated to the community at engagement events and social media. Fully anonymised data will be uploaded to a persistent DOI at the Open Science Framework; ORCID ID 00000001-9843-0778: https://osf.io/fxnwv/. Agreement with this data storage policy is included in the Consent Form. We intend to publish the results of this project in a peerreviewed journal and presented at academic conferences.

\section{Author affiliations}

${ }^{1}$ Centre for Clinical Brain Sciences, University of Edinburgh Division of Medical and Radiological Sciences, Edinburgh, UK

${ }^{2}$ Euan MacDonald Centre for Motor Neuron Disease Research, University of Edinburgh Division of Medical and Radiological Sciences, Edinburgh, UK

${ }^{3}$ Anne Rowling Regenerative Neurology Clinic, University of Edinburgh Division of Medical and Radiological Sciences, Edinburgh, UK

${ }^{4}$ Human Cognitive Neurosciences, The University of Edinburgh, Edinburgh, UK ${ }^{5}$ UK Dementia Research Institute, University of Edinburgh Division of Medical and Radiological Sciences, Edinburgh, UK

${ }^{6}$ The National Creutzfeldt-Jakob Disease Research and Surveillance Unit, Western General Hospital, Edinburgh, UK

\section{Twitter Emily Beswick @beswick_emily}

Contributors Each author has contributed significantly to one or more aspects of the study. EB, SP, SAG, JN, RD, AC, SA and SC contributed to study development and design of the protocol. In addition, EB, JN and SP will lead participant recruitment and contribute to data acquisition. EB SAG, JN, SC, SA, AC and SP drafted this work and provided critical revisions and approved the final version of this protocol. In addition, SAG provided advice on analysis plans and EB and SP made significant contributions to planned interpretation of the data. 
Funding This work was funded by a grant from MND Scotland and donations to MND-SMART SMART (Motor Neuron Disease Systematic Multi-Arm Randomised Adaptive Trial) at the Anne Rowling Regenerative Neurology Clinic and Euan MacDonald Centre for Motor Neurone Disease Research (references R45786 and D21746).

Competing interests Professor Carson is a paid editor at Journal of Neurology, Neurosurgery and Psychiatry, and gives independent testimony in Court on a range of neuropsychiatric topics.

Patient and public involvement Patients and/or the public were involved in the design, or conduct, or reporting or dissemination plans of this research. Refer to the Methods section for further details

Patient consent for publication Not required.

Provenance and peer review Not commissioned; externally peer reviewed.

Open access This is an open access article distributed in accordance with the Creative Commons Attribution Non Commercial (CC BY-NC 4.0) license, which permits others to distribute, remix, adapt, build upon this work non-commercially, and license their derivative works on different terms, provided the original work is properly cited, appropriate credit is given, any changes made indicated, and the use is non-commercial. See: http://creativecommons.org/licenses/by-nc/4.0/.

\section{ORCID iDs}

Emily Beswick http://orcid.org/0000-0001-9843-0778

Stella A Glasmacher http://orcid.org/0000-0003-1165-9153

\section{REFERENCES}

1 Leighton DJ, Newton J, Stephenson LJ, et al. Changing epidemiology of motor neurone disease in Scotland. J Neurol 2019;266:817-25.

2 Andrews JA, Jackson CE, Heiman-Patterson TD, et al. Realworld evidence of riluzole effectiveness in treating amyotrophic lateral sclerosis. Amyotroph Lateral Scler Frontotemporal Degener 2020;21:509-18.

3 van den Berg LH, Sorenson E, Gronseth G, et al. Revised airlie house consensus guidelines for design and implementation of ALS clinical trials. Neurology 2019;92:e1610-23.

4 Gul RB, Ali PA. Clinical trials: the challenge of recruitment and retention of participants. J Clin Nurs 2010;19:227-33.

5 Atassi N, Yerramilli-Rao P, Szymonifka J, et al. Analysis of startup, retention, and adherence in ALS clinical trials. Neurology 2013;81:1350-5.

6 Bedlack RS, Pastula DM, Welsh E, et al. Scrutinizing enrollment in ALS clinical trials: room for improvement? Amyotroph Lateral Scler 2008;9:257-65.

7 van Eijk RPA, Westeneng H-J, Nikolakopoulos S, et al. Refining eligibility criteria for amyotrophic lateral sclerosis clinical trials. Neurology 2019;92:e451-60.

8 Dumville JC, Torgerson DJ, Hewitt CE. Reporting attrition in randomised controlled trials. BMJ 2006;332:969-71.

9 Min J-H, Hong Y-H, Sung J-J, et al. Oral solubilized ursodeoxycholic acid therapy in amyotrophic lateral sclerosis: a randomized crossover trial. J Korean Med Sci 2012;27:200-6.

10 Beghi E, Pupillo E, Bonito V, et al. Randomized double-blind placebo-controlled trial of acetyl-L-carnitine for ALS. Amyotroph Lateral Scler Frontotemporal Degener 2013;14:397-405

11 Polit D, Hungler B. Essentials of nursing research: principles and methods. Philadelphia: Lippincott Williams \&Williams, 2001.

12 Drew A, Baird G, Baron-Cohen S, et al. A pilot randomised control trial of a parent training intervention for pre-school children with autism. Eur Child Adolesc Psychiatry 2002;11:266-72.

13 Gross D, Fogg L. Clinical trials in the 21st century: the case for participant-centered research. Res Nurs Health 2001;24:530-9.

14 Scale ALSFR. Assessment of activities of daily living in patients with amyotrophic lateral sclerosis. The ALS CNTF treatment study (ACTS) phase I-II study. GrouArch Neurol 1996;53:141-7.
15 Jaki T, Wason JMS. Multi-arm multi-stage trials can improve the efficiency of finding effective treatments for stroke: a case study. BMC Cardiovasc Disord 2018;18:215

16 Sydes MR, Parmar MKB, James ND, et al. Issues in applying multiarm multi-stage methodology to a clinical trial in prostate cancer: the MRC STAMPEDE trial. Trials 2009;10:39.

17 Ellis PM. Attitudes towards and participation in randomised clinical trials in oncology: a review of the literature. Ann Oncol 2000;11:939-46.

18 Friedman LMet al. Fundamentals of clinical trials. Vol 4. Springer, 2010.

19 Cooley ME, Sarna L, Brown JK, et al. Challenges of recruitment and retention in multisite clinical research. Cancer Nurs 2003;26:376-86.

20 Hunninghake DB, Darby CA, Probstfield JL. Recruitment experience in clinical trials: literature summary and annotated bibliography. Control Clin Trials 1987:8:6-30.

21 Madsen SM, Mirza MR, Holm S, et al. Attitudes towards clinical research amongst participants and nonparticipants. J Intern Med 2002;251:156-68.

22 Hern J. The Scottish motor neuron disease register: a prospective study of adult onset motor neuron disease in Scotland. methodology, demography and clinical features of incident cases in 1989. J Neurol Neurosurg Psychiatry 1992;55:536-41.

23 Leighton D, Newton J, Colville S, et al. Clinical audit research and evaluation of motor neuron disease (CARE-MND): a national electronic platform for prospective, longitudinal monitoring of MND in Scotland. Amyotroph Lateral Scler Frontotemporal Degener 2019;20:242-50.

24 Wicks P, Abrahams S, Masi D, et al. Prevalence of depression in a 12-month consecutive sample of patients with ALS. Eur J Neurol 2007; 14:993-1001.

25 Kessel KA, Vogel MME, Kessel C, et al. Cancer clinical trials-Survey evaluating patient participation and acceptance in a university-based Comprehensive Cancer Center (CCC). Clin Transl Radiat Oncol 2018;13:44-9.

26 Armstrong RA. When to use the B onferroni correction. Ophthalmic Physiol Opt 2014;34:502-8.

27 Crawford JR, Henry JD, Crombie C, et al. Normative data for the HADS from a large non-clinical sample. Br J Clin Psychol 2001;40:429-34.

28 Gibbons CJ, Mills RJ, Thornton EW, et al. Rasch analysis of the hospital anxiety and depression scale (HADS) for use in motor neurone disease. Health Qual Life Outcomes 2011;9:82.

29 Spielberger CD. State-Trait anxiety inventory. Corsini Encyclopedia Psychol 2010.

30 Sjonnesen K, Berzins S, Fiest KM, et al. Evaluation of the 9-item patient health questionnaire (PHQ-9) as an assessment instrument for symptoms of depression in patients with multiple sclerosis. Postgrad Med 2012;124:69-77.

31 Spitzer RL, Kroenke K, Williams JB. Validation and utility of a selfreport version of PRIME-MD: the PHQ primary care study. primary care evaluation of mental disorders. patient health questionnaire. JAMA 1999;282:1737-44

32 Felgoise SH, Feinberg R, Stephens HE, et al. Amyotrophic lateral sclerosis-specific quality of life-short form (ALSSQOL-SF): a brief, reliable, and valid version of the ALSSQOL-R. Muscle Nerve 2018;58:646-54.

33 Hennessy $\mathrm{CH}$, Moriarty DG, Zack MM, et al. Measuring healthrelated quality of life for public health surveillance. Public Health Rep 1994:109:665.

34 Radakovic R, Stephenson L, Colville S, et al. Multidimensional apathy in ALS: validation of the dimensional apathy scale. J Neurol Neurosurg Psychiatry 2016;87:663-9.

35 Radakovic R, McGrory S, Chandran S, et al. The brief dimensional apathy scale: a short clinical assessment of apathy. Clin Neuropsychol 2020;34:423-35.

36 Abrahams S, Newton J, Niven E, et al. Screening for cognition and behaviour changes in ALS. Amyotroph Lateral Scler Frontotemporal Degener 2014;15:9-14. 